\title{
Cardiovascular regulation during hypoxia in embryos of the domestic chicken Gallus gallus
}

\author{
DANE A. CROSSLEY II, ${ }^{1}$ WARREN W. BURGGREN,${ }^{1}$ AND JORDI ALTIMIRAS ${ }^{2}$ \\ ${ }^{1}$ Department of Biological Sciences, University of North Texas, Denton, Texas 76203; \\ and ${ }^{2}$ Departament of Biology, Institute of Physics and Measurement Technology, \\ Linköpings Universitet, SE-58183 Linköping, Sweden
}

Submitted 5 November 2001; accepted in final form 14 August 2002

\begin{abstract}
Crossley, Dane A. II, Warren W. Burggren, and Jordi Altimiras. Cardiovascular regulation during hypoxia in embryos of the domestic chicken Gallus gallus. Am J Physiol Regul Integr Comp Physiol 284: R219-R226, 2003. First published September 27, 2002; 10.1152/ajpregu.00654.2001. Renewed interest in the use of the embryonic chicken as a model of perinatal cardiovascular regulation has inspired new questions about the control mechanisms that respond to acute perturbations, such as hypoxia. The objectives of this study were to determine the cardiovascular responses, the regulatory mechanisms involved in those cardiovascular responses, and whether those mechanisms involved the central nervous system (CNS) of embryonic chickens. Heart rate $\left(f_{H}\right)$ and blood pressure were measured in chicken embryos of different incubation ages during exposure to different levels of hypoxia $\left(15,10\right.$, and $\left.5 \% \mathrm{O}_{2}\right)$. At all levels of hypoxia and at all developmental ages, a depression of $\mathrm{f}_{\mathrm{H}}$ and arterial pressure was observed, with the exception of day 20 embryos in 15 and $10 \%$ $\mathrm{O}_{2}$. The intensity of the embryonic $\mathrm{f}_{\mathrm{H}}$ and blood pressure responses were directly related to the level of hypoxia used. Muscarinic and $\alpha$-adrenergic receptor stimulation limited the hypoxic hypotension on days 15-19 and 15-21, respectively, as indicated after blockade with atropine and phentolamine. During the final 3 days of incubation, the intensity of the hypoxic hypotension was magnified due to $\alpha$-vasodilation caused by $\beta$-adrenergic and muscarinic receptor stimulation. In 19- to 21-day-old embryos, the $\mathrm{f}_{\mathrm{H}}$ response to hypoxia was limited by $\alpha$-adrenergic receptor stimulation as indicated by the accentuated bradycardia after blockade with phentolamine. Furthermore, on day 21, atropine limited the hypoxic bradycardia, indicating that muscarinic receptors also play a role in the $\mathrm{f}_{\mathrm{H}}$ response at this age. In addition, the muscarinic actions on the heart and the adrenergic effects on the vasculature appeared to occur through a hypoxic-induced direct release from chromaffin tissue and autonomic nerve terminals. Thus, in embryonic chickens, the only cardiovascular response to hypoxia that involves the CNS was the cholinergic regulation of arterial pressure after day 15 of incubation. Therefore, although embryonic chickens and fetal sheep, the standard models of perinatal cardiovascular physiology, respond to hypoxia with a similar redistribution of cardiac output, the underlying mechanisms differ between these species.
\end{abstract}

catecholamine; autonomic; adrenergic; muscarinic; perinatal hypoxia

EMBRYONIC CHICKENS EXPOSED to hypoxia show an $\alpha$-adrenergic-mediated redistribution of cardiac output

Address for reprint requests and other correspondence: D. A. Crossley II, Dept. of Ecology and Evolutionary Biology, Univ. of California at Irvine, Irvine, CA 92697 (E-mail: dcrossle@uci.edu). with the preferential perfusion of the brain, heart, and chorioallantoic membrane (CAM; 18, 20). This pattern of hypoxic redistribution of cardiac output is similar to that seen in fetal sheep, which is known to accompany redistribution of blood flow with reflexive changes in heart rate and peripheral resistance (12). In the sheep fetus, the neural reflex, a vagal-mediated bradycardia, and an $\alpha$-adrenergic efferent-mediated hypertension are followed by numerous endocrine responses $(12,13)$. However, recent findings in embryonic chickens suggest that cardiovascular regulation at the time of hatching is less developed in chickens than in sheep at birth. This evidence includes the absences of vagal tone throughout the prenatal period in chickens (10), the late (day 19) appearance of baroreflexive responses (1), and the hypotension displayed when exposed to hypoxia $(10,24)$. These three characteristics of chicken development differ from those found in fetal sheep during a similar period of gestation in response to similar experimental conditions $(11,23)$.

Therefore it was hypothesized that embryonic chickens would rely on endocrine control mechanisms during acute periods of hypoxia. Furthermore, given the importance of adrenergic receptor stimulation in maintaining normal cardiovascular function in embryonic chickens (10), we also hypothesized that this would be the primary mechanism for regulating blood pressure and heart rate during hypoxic challenges (20). Thus the goal of this study was to determine the heart rate and arterial pressure responses, the regulatory mechanisms involved in those responses, and the role of the autonomic nervous system in regulating cardiovascular function during hypoxia in embryonic chickens.

\section{MATERIAL AND METHODS}

Subjects of study. Freshly laid chicken eggs, Gallus gallus (White Leghorn strain), were purchased from University of Texas A\&M and shipped overnight to the University of North Texas, Department of Biological Sciences. On arrival, eggs were placed in incubation at $38 \pm 0.5^{\circ} \mathrm{C}, 60-70 \%$ relative humidity and turned automatically every $3 \mathrm{~h}$.

The costs of publication of this article were defrayed in part by the payment of page charges. The article must therefore be hereby marked "advertisement" in accordance with 18 U.S.C. Section 1734 solely to indicate this fact. 
Surgical procedures. Before each experimental series, eggs were removed from the incubator, candled to locate a chorioallantoic artery, and placed in a holder thermostatically controlled at $38 \pm 0.5^{\circ} \mathrm{C}$. A $1-\mathrm{cm}^{2}$ portion of the eggshell was then removed, exposing the previously located artery. This artery was then occlusively catheterized with heat-pulled PE-90 tubing filled with heparinized $0.9 \%$ saline under a dissection microscope (Wild M3Z). Once the catheter was in place, it was fixed to the shell with cyanoacrylic glue. Subsequently, the egg was placed in the experimental chamber that consisted of a water-jacketed glass container fitted with a glass lid. The lid had three ports, providing an avenue for externalizing the arterial catheter as well as routes for inflow and outflow of different gas mixtures. During the experiments, all eggs were maintained at $38 \pm 0.5^{\circ} \mathrm{C}$ in watersaturated air.

Signal recording and calibration. The arterial catheter from each egg was attached to a pressure transducer (WPI, type BLPR), and this was connected to a bridge amplifier (CB Sciences, model ETH-400). Pressure signals were stored in a computer using PowerLab data-acquisition software. Heart rate was continuously calculated from the pressure signal via an acquisition software tachograph. Reference zero pressure was set at the top of the experimental bath, and all values were corrected after the experiment as previously described (1).

Experimental protocol. The study consisted of six different experimental series. The number of embryos used in each series at each incubation age is indicated in Table 1. All embryos were only used in one experimental series. All series began with a control period of 30 min postsurgery to allow blood pressure and heart rate to reach stable values. Embryos that failed to do so were removed from the study. For all series that involved pharmacological manipulations, drugs were administered via a $\mathrm{T}$ connector in the arterial catheter line. Each drug injection was followed by a saline flush with double the volume of the drug solution. Total injection volumes were always $<5 \%$ of the total blood volume. This volume had no significant effect on cardiovascular function as previously reported (1). For all experimental series, 20-day-old embryos were defined as internally pipped eggs verified by candling. Twenty-one-day-old embryos were defined as embryos externally pipped. The sequence of experimental series described represents the flow of the investigation from the general hypoxic response to the determination of the receptors involved and then the contribution of any reflexive responses.

In series $I$, cardiovascular responses were examined in embryos at days $9,12,15,18,19,20$, and 21 of a 21-day incubation period. Each developmental group was exposed to step changes in ambient oxygen composition $(15,10$, and $5 \%$

Table 1. Number of embryos used in each of the experimental series on each experimental day

\begin{tabular}{|c|c|c|c|c|c|c|c|c|}
\hline \multirow[b]{2}{*}{ Series } & \multirow[b]{2}{*}{ Description } & \multicolumn{7}{|c|}{ Day of Incubation } \\
\hline & & 9 & 12 & 15 & 18 & 19 & 20 & 21 \\
\hline$I$ & hypoxia: $15 \%, 10 \%, 5 \% \mathrm{O}_{2}$ & 6 & 7 & 7 & 8 & 6 & 5 & 5 \\
\hline II & $\begin{array}{l}\text { autonomic blockade during } \\
10 \% \mathrm{O}_{2}\end{array}$ & 一 & 5 & 6 & 7 & 7 & 7 & 7 \\
\hline III & $\begin{array}{l}\text { catecholamine release during } \\
10 \% \mathrm{O}_{2}\end{array}$ & 一 & 一 & - & 4 & 6 & 5 & 6 \\
\hline$I V$ & effect of tyramine & - & 5 & 5 & 5 & 8 & 5 & 5 \\
\hline$V$ & $\begin{array}{l}\text { sympathectomy during } 10 \% \\
\mathrm{O}_{2}\end{array}$ & - & 一 & 5 & 5 & 6 & 5 & 5 \\
\hline$V I$ & $\begin{array}{l}\text { ganglionic blocker during } \\
10 \% \mathrm{O}_{2}\end{array}$ & 一 & 5 & 5 & 5 & 5 & 5 & 5 \\
\hline
\end{tabular}

$\mathrm{O}_{2}$ ) for $5 \mathrm{~min}$, with a 30 -min to 1 -h recovery period as dictated by the return of cardiovascular variables to prehypoxic values. All gas mixtures were set using two gas flow rotameters (Cole Parmer) for oxygen and nitrogen.

In series II, the effects of autonomic receptor antagonists on the hypoxic cardiovascular response were determined in a sequential manner. Embryonic chickens at days 12, 15, 18, 19,20 , and 21 were used. After a 30-min control period, embryos were exposed to $10 \% \mathrm{O}_{2}$ for $5 \mathrm{~min}$ and then returned to normoxia for $60 \mathrm{~min}$. This initial exposure was followed by an injection of the cholinergic antagonist atropine $(1 \mathrm{mg} / \mathrm{kg})$. Pressure and heart rate were allowed to stabilize (average stabilization time of $45 \mathrm{~min}$ ) before a new recording period consisting of 30 min control +5 min hypoxia $\left(10 \% \mathrm{O}_{2}\right)$ was taken. This recording protocol was serially repeated in the same embryos with the $\beta$-adrenergic antagonist propranolol ( $3 \mathrm{mg} / \mathrm{kg}$ ) and the $\alpha$-adrenergic antagonist phentolamine (1 $\mathrm{mg} / \mathrm{kg}$ ). The antagonists were always given in the same order with a stabilization period after the injection of 30-60 min.

In series III, embryos were catheterized on days 18, 19, 20, and 21 of incubation and allowed $30 \mathrm{~min}$ to reach control blood pressures and heart rates. Subsequently, a control blood sample $(200 \mathrm{ml})$ was taken from the arterial catheter and immediately spun down to separate the plasma. The erythrocytes were then suspended in $0.9 \%$ saline to the original volume, withdrawn, and injected back into the embryo via the arterial catheter. Plasma samples were then mixed with $5 \mu \mathrm{l}$ of an EGTA-glutathione solution $(0.2 \mathrm{M}-0.2$ $\mathrm{M})$ to prevent catecholamine oxidation. A second blood sample $(200 \mathrm{ml})$ was taken after a 5 -min exposure to $10 \% \mathrm{O}_{2}$ and prepared as described. Plasma epinephrine and norepinephrine concentrations in the normoxic and hypoxic samples were determined using HPLC techniques previously described by the authors (10).

Series $I V$ and $V$ were carried out to distinguish between neural and humoral catecholamine actions during hypoxia. This differentiation was accomplished using chemical sympathectomy with 6-hydroxydopamine (6-OH). In fetal sheep, sympathectomy is carried out over a period of days (serial injections) to ensure the total destruction of sympathetic terminals (4). In this series, tyramine was used as a selective norepinephrine-releasing agent (29). Injections before and after treatment with 6-OH were used to test for the presence of functional sympathetic nerve terminals and to verify the degree of chemical sympathectomy achieved 60 min after a bolus injection of $6-\mathrm{OH}$. Thus, in series $I V$, after the catheterization and a 30-min control period, a single injection of tyramine $(10 \mathrm{mg} / \mathrm{kg})$ was given to embryonic chickens on days $12,15,18,19,20$, and 21 of incubation. After a 30-min recovery period, chemical sympathectomy was conducted with $6-\mathrm{OH}(20 \mathrm{mg} / \mathrm{kg})$ only on days $15,18,19,20$, and 21 of incubation because tyramine showed no effects on day 12 . After $60 \mathrm{~min}$, the injection of $6-\mathrm{OH}$ was followed by a second administration of tyramine $(10 \mathrm{mg} / \mathrm{kg})$ for comparisons of the cardiovascular response to tyramine before and after $6-\mathrm{OH}$ treatment.

In series $V$, after a 30-min control period, chemical sympathectomy with $6-\mathrm{OH}(20 \mathrm{mg} / \mathrm{kg})$ was carried out as in series $I V$ in embryos on days 15, 18, 19, 20, and 21 of incubation. After a 60-min recovery period, 6-OH-treated embryos were exposed to hypoxia $\left(10 \% \mathrm{O}_{2}\right)$, and cardiovascular response was recorded as in series II.

In series VI, after the surgical recovery period (30 $\mathrm{min}$ ), embryonic chickens at days 15, 18, 19, 20, and 21 were exposed to a 5-min hypoxic $\left(10 \% \mathrm{O}_{2}\right)$ period. A recovery period was then allowed (60 $\mathrm{min})$ before an injection of the ganglionic blocking agent hexamethonium $(25 \mathrm{mg} / \mathrm{kg})$ was 

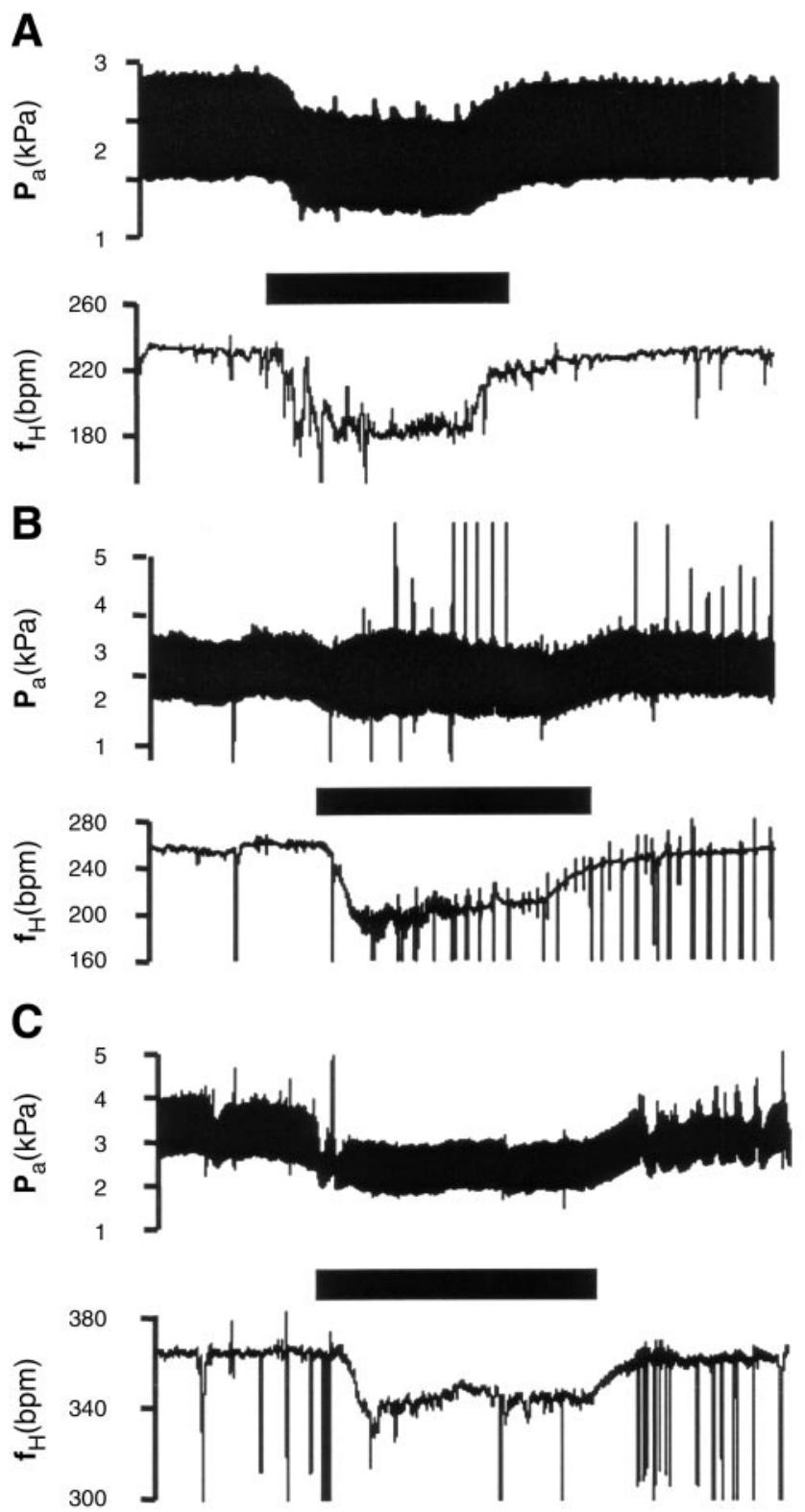

Fig. 1. Representative traces depicting effects of $10 \% \mathrm{O}_{2}$ on arterial pressure $\left(\mathrm{P}_{\mathrm{a}}\right)$ and heart rate $\left(\mathrm{f}_{\mathrm{H}}\right)$ in embryos of $19(A), 20(B)$, and 21 $(C)$ days of incubation. Thick bars over traces indicate the duration of the hypoxic exposure. bpm, Beats/min.

conducted. After the injection, arterial pressure and heart rate stabilized within $30 \mathrm{~min}$. This was followed by a second 5 -min hypoxic $\left(10 \% \mathrm{O}_{2}\right)$ exposure and recovery.

At the completion of all series, embryos were euthanized with an overdose of pentobarbital sodium and $\mathrm{KCl}$. The eggs were then frozen to determine the heart position needed for correction of the blood pressure values (1).

Statistical analysis. A matched-pairs Wilcoxon nonparametric test was used to assess statistical differences in heart rate and blood pressure before and after exposure to hypoxia with or without drugs. A $U$-Mann-Whitney comparison nonparametric test was conducted between adjacent days of incubation to determine changes in the cardiovascular response to $10 \% \mathrm{O}_{2}$ as well as the responses to various blocking agents. A Bonferroni correction was applied for data used more than once in a given analysis. All data are presented as means $\pm \mathrm{SE}$.

\section{RESULTS}

Series I: effects of hypoxia. Chicken embryos responded to all levels of hypoxia $\left(15,10\right.$, and $\left.5 \% \mathrm{O}_{2}\right)$ with a significant $(P<0.05)$ hypotensive bradycardia up to day 19 of incubation. This pattern differed on days 20 and 21 of incubation as illustrated by the representative traces in Fig. 1. Before day 20 of incubation, changes in mean arterial pressure (MAP) and heart rate were related to the severity of the hypoxic challenge, with significant differences between hypoxic responses. MAP dropped an average of $12 \%\left(15 \% \mathrm{O}_{2}\right)$, $18 \%\left(10 \% \mathrm{O}_{2}\right)$, and $26 \%\left(5 \% \mathrm{O}_{2}\right)$, whereas heart rate dropped an average of $9 \%\left(15 \% \mathrm{O}_{2}\right), 19 \%\left(10 \% \mathrm{O}_{2}\right)$, and $34 \%\left(5 \% \mathrm{O}_{2}\right)$ during this period of study (Fig. 2). On day 20 there was no pressure response to $15 \%$ and $10 \% \mathrm{O}_{2}$, but $5 \% \mathrm{O}_{2}$ resulted in a drop in MAP and heart rate. Day 21 embryonic chickens reverted to the hypoxic hypotensive bradycardia to all levels of hypoxia shown in earlier days of incubation $(P<0.05)$.

Series II: hypoxic responses after muscarinic and adrenergic antagonists. The cardiovascular responses to muscarinic as well as $\alpha$ - and $\beta$-adrenergic blockade were similar to those previously described (10) and will not be discussed further.

Atropine enhanced the hypoxic hypotension on days 15, 18, and 19 (Fig. 3). However, it had no effect on the hypoxic MAP response on days 12 and 20 (Fig. 3). Furthermore, atropine reduced the hypoxic hypoten-
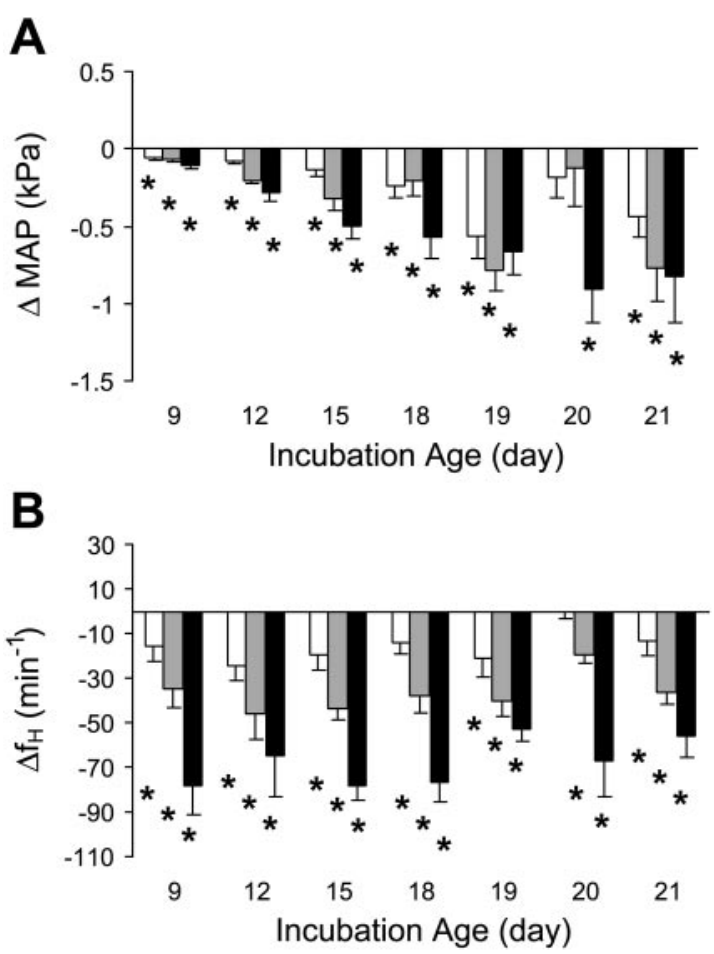

Fig. 2. Effects of $15 \% \mathrm{O}_{2}$ (open bars), $10 \% \mathrm{O}_{2}$ (shaded bars), and $5 \%$ $\mathrm{O}_{2}$ (solid bars) on mean arterial pressure (MAP) change $(A)$ and $\mathrm{f}_{\mathrm{H}}$ change $(B)$ at different days of chicken development. Data are means \pm SE. $*$ Significant differences from control $(P<0.05)$. 
A

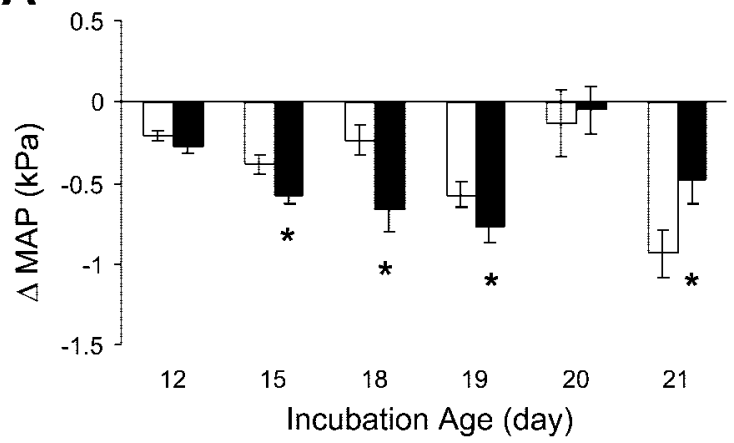

B

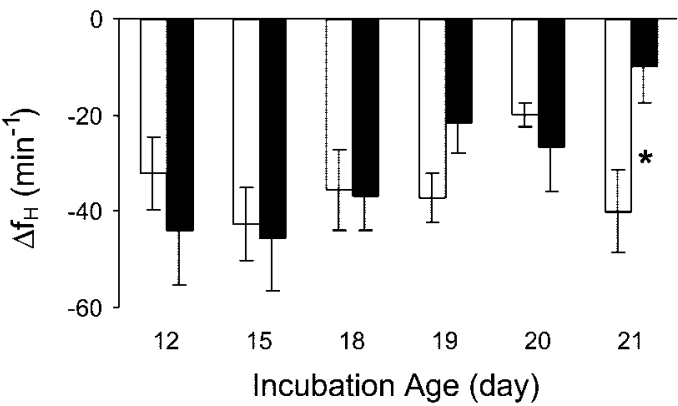

Fig. 3. Effects of $10 \% \mathrm{O}_{2}$ before (open bars) and after (solid bars) the administration of atropine on $\operatorname{MAP}(A)$ and $\mathrm{f}_{\mathrm{H}}(B)$ at different days of chicken development. ${ }^{*}$ Significant differences from control hypoxic exposure $(P<0.05)$.

sion on day 21 of incubation $(P<0.05$; Fig. 3). In addition, atropine changed the embryonic hypoxic bradycardia on day 21 of incubation, only reducing the response from a control hypoxic bradycardia of $40 \pm 9$ $\min ^{-1}$ to a postatropine hypoxic bradycardia of $10 \pm 6$ $\min ^{-1}(P<0.05$; Fig. 3$)$.

Propranolol injection after atropine had significant effects on the MAP response to hypoxia during the last 3 days of incubation (Fig. $4 A$ ). The hypoxic hypotension was significantly reduced $(P<0.05)$ on day 19 of incubation (from a $0.76 \pm 0.10 \mathrm{kPa}$ drop to a postpropranolol hypoxic drop of $0.10 \pm 0.15 \mathrm{kPa}$ ). On days 20 and 21 of incubation, the injection of propranolol resulted in a reversal of the MAP response to hypoxia from a hypotension to a hypertension with MAP increases during hypoxia of $0.63 \pm 0.13$ and $0.65 \pm 0.23$ $\mathrm{kPa}$, respectively (Fig. 4A). No significant differences in the hypoxic heart rate response were observed after propranolol injection (Fig. $4 B$ ).

Embryonic chickens exposed to $10 \% \mathrm{O}_{2}$ after complete autonomic blockade (atropine + propranolol + phentolamine) exhibited opposite MAP responses to those seen during hypoxia after atropine and propranolol injection (Fig. 4C). $\alpha$-Blockade produced a significant $(P<0.05)$ decrease in MAP during hypoxia compared with the response after atropine and propranolol injection on days 15, 19, 20, and 21 of incubation (Fig. $4 C$ ). The average hypoxic change in MAP after phentolamine increased with development: $0.06 \mathrm{kPa}$ on day $12,0.21 \mathrm{kPa}$ on day $15,0.26 \mathrm{kPa}$ on day $18,0.74 \mathrm{kPa}$ on day $19,1.44 \mathrm{kPa}$ on day 20 , and $1.66 \mathrm{kPa}$ on day 21 . In addition, during the final 3 days of incubation, phentolamine resulted in an increased hypoxic bradycardia that was significantly different $(P<0.05)$ from that of embryos treated with atropine and propranolol alone on days 19 and 20 (Fig. 4D). Large differences between embryos on day 21 may account for the nonsignificant change in hypoxic heart rate response on this day of incubation $(P<0.06)$ (Fig. $4 D)$.

Series III: catecholamine release during hypoxia. A marked release of catecholamines occurred during exposure to $10 \% \mathrm{O}_{2}$ in day 18 embryos and older (Table 2). Norepinephrine release peaked on day 19 (a 16-fold

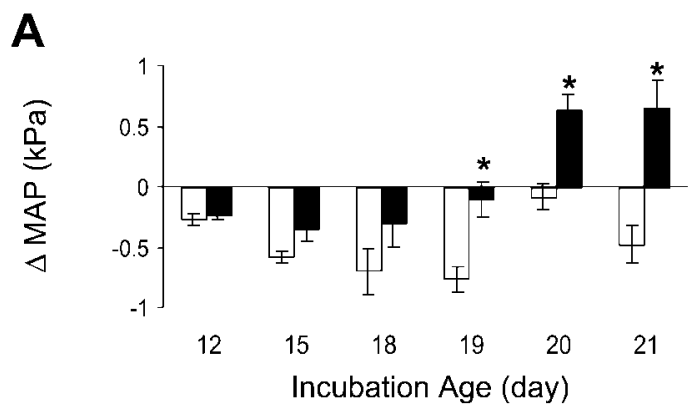

B
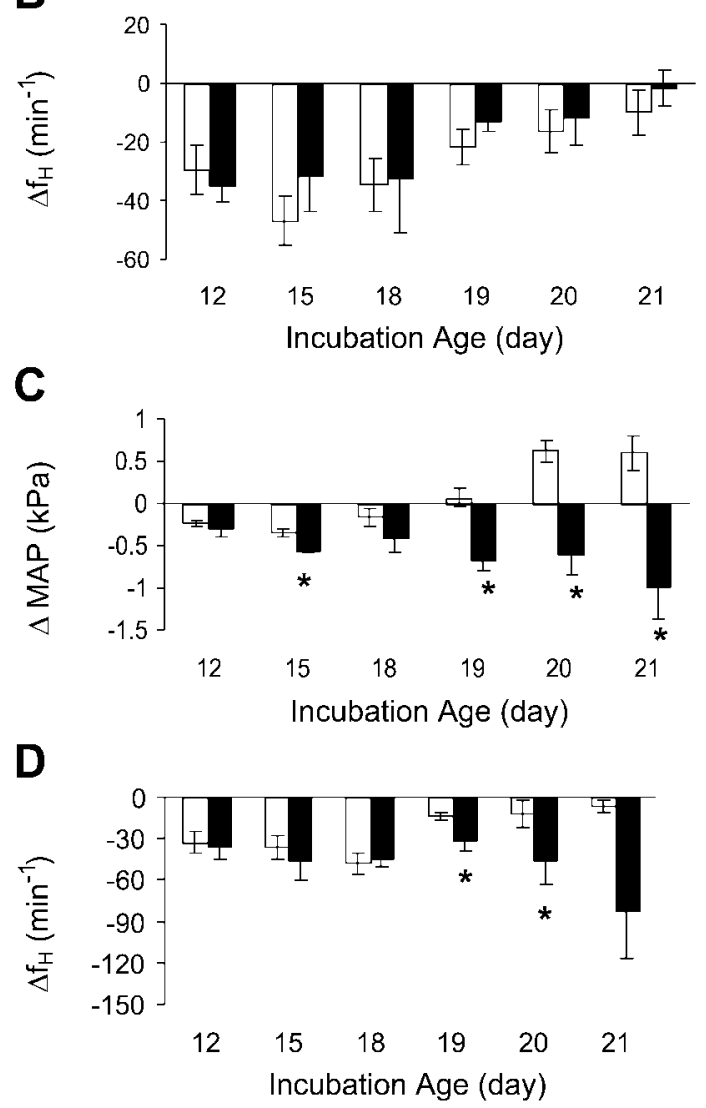

Fig. 4. $A$ and $B$ : effects of $10 \% \mathrm{O}_{2}$ before (open bars) and after (solid bars) the administration of propranolol on MAP $(A)$ and $f_{H}(B)$ at different days of chicken development. $C$ and $D$ : effects of $10 \% \mathrm{O}_{2}$ before (open bars) and after (solid bars) the administration of phentolamine on MAP $(C)$ and $f_{H}(D)$ at different days of chicken development. Data are means $\pm \mathrm{SE}$. * Significant differences from control hypoxic exposure $(P<0.05)$. 
Table 2. Percentage increase above control plasma levels of Epi and $\mathrm{NE}$ during an exposure to $10 \% \mathrm{O}_{2}$

\begin{tabular}{crccc}
\hline \hline Day & \multicolumn{1}{c}{$\%$ Epi } & $P$ & $\% \mathrm{NE}$ & $P$ \\
\hline 18 & $536 \pm 153$ & 0.067 & $824 \pm 520$ & 0.067 \\
19 & $535 \pm 129$ & 0.028 & $1,592 \pm 608$ & 0.028 \\
20 & $1,469 \pm 780$ & 0.043 & $445 \pm 99$ & 0.043 \\
21 & $1,139 \pm 571$ & 0.046 & $439 \pm 240$ & 0.028 \\
\hline
\end{tabular}

Data are presented as means \pm SE. $P$ indicates the significance of the percentage change. Epi, epinephrine; NE, norepinephrine.

increase above normoxic levels), whereas epinephrine release was maximal on day 20 (a 15 -fold increase).

Series IV and V: hypoxic response after chemical sympathectomy. A single administration of tyramine (series IV) induced a significant hypertension in embryonic chickens at day 15 of incubation and older, producing an average elevation in pressure ranging from 0.16 to $0.75 \mathrm{kPa}$ (Table 3). In addition, day 19 and 21 embryos showed a significant tachycardic response to tyramine injection (Table 3 ).

The response to tyramine was not eliminated with the $6-\mathrm{OH}$ administration regimen used (Table 4), but the hypertensive and tachycardic responses to tyramine were reduced. The different pressure and heart rate responses to tyramine before and after chemical sympathectomy were significant in day 19 embryos only (Table 4).

In series $V, 6-\mathrm{OH}$ (administered as in series $I V$ ) magnified the hypoxic pressure response by enhancing the hypoxic hypotension on day 20 (from a $0.0 \pm 0.14$ $\mathrm{kPa}$ change before $6-\mathrm{OH}$ to a $0.52 \pm 0.05 \mathrm{kPa}$ pressure drop after 6-OH; Table 5). Furthermore, an enhanced hypoxic bradycardia was evident in embryos at days 19 and 20 after $6-\mathrm{OH}$ treatment (a $24 \%$ and $60 \%$ greater hypoxic bradycardia, respectively; Table 5).

Series VI: hypoxic response after ganglionic blockade. Hexamethonium injections triggered changes in MAP and heart rate that were similar to those previously shown over the same period of chicken incubation (10). The hypoxic response posthexamethonium was significantly different during the late stages of embryonic development (Fig. 5). An enhancement of the hypoxic hypotension was observed on days 18 (a $31 \%$ greater pressure drop) and 19 (a 84\% greater pressure drop), with no difference on day 20. On day 21, the effects of hexamethonium were reversed compared with previous days

Table 3. Effects of tyramine $(10 \mathrm{mg} / \mathrm{kg})$ on $M A P$ and $f_{H}$

\begin{tabular}{ccclll}
\hline \hline & \multicolumn{2}{c}{ MAP, $\mathrm{kPa}$} & & \multicolumn{2}{c}{$\mathrm{f}_{\mathrm{H}, \mathrm{min}^{-1}}$} \\
\cline { 2 - 3 } \cline { 5 - 6 } Day & Control & Tyramine & & Control & Tyramine \\
\hline 12 & $1.36 \pm 0.11$ & $1.38 \pm 0.10$ & & $207 \pm 10$ & $209 \pm 11$ \\
15 & $2.22 \pm 0.25$ & $2.38 \pm 0.24^{*}$ & & $226 \pm 18$ & $240 \pm 14$ \\
18 & $2.30 \pm 0.13$ & $2.83 \pm 0.25^{*}$ & & $229 \pm 8$ & $241 \pm 7$ \\
19 & $2.77 \pm 0.14$ & $3.20 \pm 0.15^{*}$ & & $237 \pm 6$ & $258 \pm 6^{*}$ \\
20 & $2.98 \pm 0.12$ & $3.64 \pm 0.23^{*}$ & & $268 \pm 7$ & $279 \pm 7$ \\
21 & $3.91 \pm 0.35$ & $4.66 \pm 0.48^{*}$ & & $294 \pm 17$ & $308 \pm 15^{*}$ \\
\hline
\end{tabular}

Data are presented as absolute values (means $\pm \mathrm{SE}) . *$ Significant difference from control $(P<0.05)$. MAP, mean arterial pressure; $f_{\mathrm{H}}$, heart rate.
Table 4. Effects of tyramine $(10 \mathrm{mg} / \mathrm{kg}$ ) on MAP and $f_{H}$ before and after chemical sympathectomy with 6-hydroxydopamine (20 $\mathrm{mg} / \mathrm{kg}$ )

\begin{tabular}{cccccc}
\hline \hline & \multicolumn{2}{c}{$\mathrm{MAP}, \mathrm{kPa}$} & & \multicolumn{2}{c}{$\mathrm{f}_{\mathrm{H}, \mathrm{min}^{-1}}$} \\
\cline { 2 - 3 } \cline { 5 - 6 } Day & Before 6-OH & After 6-OH & & Before 6-OH & After 6-OH \\
\hline 15 & $1.68 \pm 0.40$ & $0.85 \pm 0.28$ & & $14 \pm 6$ & $4 \pm 5$ \\
18 & $5.34 \pm 1.73$ & $2.54 \pm 0.52$ & & $11 \pm 4$ & $12 \pm 3$ \\
19 & $4.33 \pm 0.38$ & $2.56 \pm 0.39 *$ & & $21 \pm 2$ & $13 \pm 3^{*}$ \\
20 & $6.76 \pm 2.04$ & $4.47 \pm 0.80$ & & $10 \pm 5$ & $5 \pm 3$ \\
21 & $7.69 \pm 1.78$ & $6.19 \pm 1.56$ & & $15 \pm 4$ & $12 \pm 5$ \\
\hline
\end{tabular}

Data are presented as differences between control and posttyramine injection (means $\pm \mathrm{SE}$ ). * Significant effect of 6 -hydroxydopamine $(6-\mathrm{OH})(P<0.05)$.

with a reduction in the hypoxic hypotension after hexamethonium (72\% reduction in the pressure drop; Fig. 5). These changes in arterial pressure to $10 \% \mathrm{O}_{2}$ after hexamethonium were not accompanied by differences in the heart rate response to hypoxia (Fig. 5).

\section{DISCUSSION}

Throughout the second half of incubation, embryonic chickens of the White Leghorn strain exhibited a clear depression of heart rate and arterial pressure when exposed to hypoxia as previously reported (24). The intensity of this response was directly related to the intensity of the hypoxic exposure, with the largest effects observed in all embryos that were exposed to $5 \% \mathrm{O}_{2}$ (Fig. 2). Early in development (day 12 of incubation), the hypoxic bradycardia appeared to be primarily caused by the direct action of low $\mathrm{O}_{2}$ on the cardiac muscle. By the end of the incubation, this response appeared also to be due to stimulation of cholinergic receptors (day 21). In addition, the hypoxic hypotension found in embryonic chickens was limited by both an $\alpha$-adrenergic- and a cholinergic receptor-stimulated vasoconstriction from days 15 to 19 in embryonic chickens. During the final 3 days of incubation, a third regulatory element was present, namely a $\beta$-adrenergic-stimulated vasodilator tone. At the same time, a reversal of the cholinergic response (from vasoconstrictor to vasodilator) appeared on day 21 . The pharmacological evidence suggests that the cholinergic receptor stimulated action on pressure during hypoxia originated above the ganglionic level. Conversely, the adrenergic receptor-stimulated pressure changes were

Table 5. Absolute change in blood pressure and $f_{H}$ during a 5-min exposure to $10 \% \mathrm{O}_{2}$ before and after the administration of 6-hydroxydopamine $(20 \mathrm{mg} / \mathrm{kg})$

\begin{tabular}{cccccc}
\hline \hline & \multicolumn{2}{c}{ MAP, $\mathrm{kPa}$} & & \multicolumn{2}{c}{$\mathrm{f}_{\mathrm{H}, \mathrm{min}^{-1}}$} \\
\cline { 2 - 3 } \cline { 5 - 6 } Day & Before 6-OH & After 6-OH & & Before 6-OH & After 6-OH \\
\hline 15 & $-0.37 \pm 0.03$ & $-0.3 \pm 0.03$ & & $-42 \pm 10$ & $-44 \pm 8$ \\
18 & $-0.46 \pm 0.1$ & $-0.28 \pm 0.1$ & & $-30 \pm 10$ & $-50 \pm 6$ \\
19 & $-0.41 \pm 0.07$ & $-0.43 \pm 0.07$ & & $-38 \pm 5$ & $-47 \pm 5^{*}$ \\
20 & $0 \pm 0.14$ & $-0.52 \pm 0.14^{*}$ & & $-39 \pm 9$ & $-61 \pm 12^{*}$ \\
21 & $-0.42 \pm 0.1$ & $-0.67 \pm 0.24$ & & $-37 \pm 8$ & $-39 \pm 8$ \\
\hline
\end{tabular}

$*$ Data are presented as mean $\pm \mathrm{SE}$. Significant changes $(P<0.05)$ in MAP and $\mathrm{f}_{\mathrm{H}}$. 


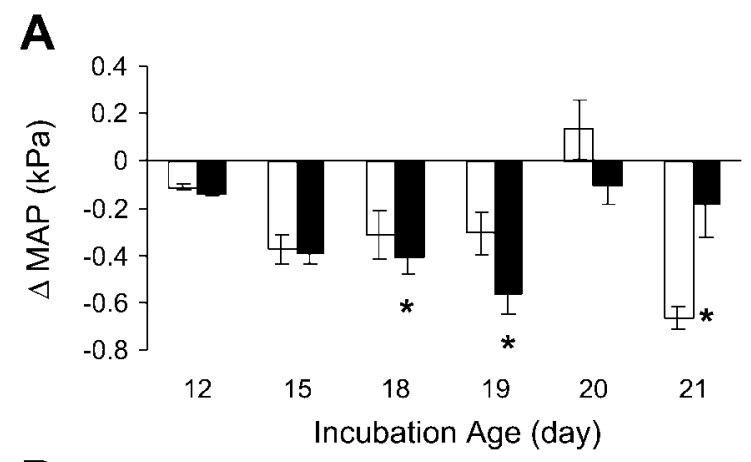

B

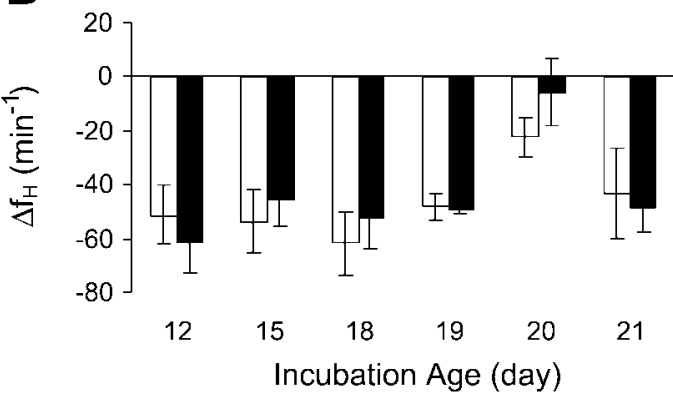

Fig. 5. Effect of $10 \% \mathrm{O}_{2}$ on $\mathrm{MAP}(A)$ and $\mathrm{f}_{\mathrm{H}}(B)$ before (open bars) and after (solid bars) hexamethonium administration at different days of chicken development. Data are means \pm SE. * Significant differences from control hypoxic exposure $(P<0.05)$.

predominantly derived from the humoral catecholamine response, with a neural contribution during the last days of incubation. Thus, in embryonic chickens, hypoxia appeared to directly stimulate chromaffin tissue and sympathetic nerve terminals to release catecholamines. This then stimulates adrenergic receptors, causing changes in heart rate and arterial pressure. Furthermore, the cholinergic receptor-stimulated changes in arterial pressure alone originated above the ganglionic level, possibly indicating a reflexive mechanism controlling pressure in embryonic chickens.

Effects of hypoxia in embryos up to 18 days of incubation. Until day 18 of incubation, the hypoxic bradycardia evident in embryonic chickens was not due to a reflexive (neural) response. This statement is based on the persistent hypoxic bradycardia after ganglionic blockade with hexamethonium (Fig. 5). In addition, the cholinergic and adrenergic receptor stimulation contributed little to the chronotropic response to hypoxia in embryos as was evident in the persistent hypoxic bradycardia after $\beta$ - and $\alpha$-adrenergic blockade (Fig. 4). Therefore, at these early developmental stages $(\leq 18$ days), the most relevant finding was the existence of a muscarinic-dependent regulation of systemic arterial pressure during hypoxia. Such a cholinergic vasomotor control is absent in mammals but is present in adult birds (3). Thus, on day 15, cholinergic receptors are involved in a vasoconstrictor response during hypoxia, lessening the severity of the hypoxic hypotension.

During hypoxia, the two potential routes for cholinergic receptor stimulation are the release of $\mathrm{ACh}$ from local vascular or neural sources. These two nonexclud- ing alternatives were addressed by comparing the results from series II (atropine) and series VI (hexamethonium). On day 15, local release was the most feasible mechanism, because the hypoxic hypotension was enhanced only after a muscarinic blockade at the target organ but not when neural transmission was blocked with hexamethonium. A local release of ACh in response to different stimuli by vascular endothelial cells has been previously demonstrated in numerous adult species $(6,16)$ and may relate to the findings here in embryonic chickens. As the embryo developed beyond day 15, the hypoxia pressure response became similar after both cholinergic and ganglionic blockade (Figs. $3 A$ and $5 A$ ). This indicates that the pressure changes that accompanied hypoxia were mediated via an increase in neural activity above the ganglionic level. Thus embryonic chickens may possess a functional central reflex that changes arterial pressure during hypoxia.

Effects of hypoxia in embryos older than 18 days. Compared with earlier days of incubation, cardiovascular regulation during the last 3 days of chicken incubation became complex. This increased complexity was caused by the appearance of a muscarinic, $\alpha$-adrenergic, and $\beta$-adrenergic receptor stimulation action on the heart as well as the vasculature during hypoxia. Therefore, these receptors may be paramount to maintaining cardiovascular function in the chicken through the late perinatal and hatching periods.

Both mechanical and regulatory mechanisms may contribute to the observed changes in the hypoxic response after blockade in embryos older than day 18. For instance, complete autonomic blockade (phentolamine preceded by atropine and propranolol) accentuated the hypoxic hypotensive and bradycardic response (Fig. 4). The hypotension and bradycardia are likely coupled, given that the large drop in arterial pressure may compromise venous return, increasing filling time and decreasing heart rate. However, a positive chronotropic effect of $\alpha$-adrenoceptors on the heart cannot be excluded as previously reported in fetal lambs (8). If a positive $\alpha$-adrenergic stimulation of heart rate occurred during hypoxia it was likely due to the direct hypoxic induced release of norepinephrine from sympathetic terminals. This is based on the chemically sympathectomized embryos, which displayed a greater hypoxic bradycardia on day 19 and 20 of chicken incubation (Table 5 ). Given that $\beta$-blockade did nothing to the hypoxic heart rate response (Fig. $4 B$ ), the adrenergic limitation of the bradycardic intensity must originate from $\alpha$-adrenergic stimulation. Furthermore, that the hypoxic bradycardia was magnified after partial sympathectomy (Table 5) but not after hexamethonium (Fig. 5) suggests that the adrenergic receptor stimulation of the heart was caused by a direct hypoxic-induced release of catecholamines from the sympathetic nerve terminals. This type of nonreflexive hypoxic heart rate response was also present in externally pipped (day 21) embryos.

In externally pipped embryos ( 21 days), hypoxic bradycardia could be eliminated via muscarinic blockade 
with atropine (Fig. 2) but not via ganglionic blockade with hexamethonium (Fig. 5), indicating that the cholinergic response on day 21 was not reflexive. The effects of atropine on day 21 contrast with those of a prior study (10) and could be attributed to the lessprecise determination of embryonic age in an earlier study by the authors. However, when combined, the atropine and hexamethonium data indicate that the hypoxic depression of heart rate in embryonic chickens occurs without an increase in vagal activity and thus was due to the direct effects of hypoxia.

As previously reported, tonic regulation of the total embryonic chicken vasculature (embryonic plus extraembryonic: yolk sac and chorioallantois) is based on a $\beta$-adrenergic vasodilation superimposed on an $\alpha$-adrenergic vasoconstriction (10). During hypoxia the $\alpha$-adrenergic tone was elevated, limiting the fall in CAM arterial pressure in embryos during the last third of incubation (Fig. 4). On days 15 and 18, however, the $\alpha$-adrenergic stimulation was insufficient to offset the overriding hypoxic reduction in arterial pressure. Later (days 19-21) the $\alpha$-adrenergic response was sufficient to cause a general hypoxic hypertension that is overridden by a $\beta$-adrenergic vasodilation (Fig. 4 ). The main site of $\beta$-adrenergic vasodilation appeared to be the chorioallantoic vascular bed, as shown in preliminary experiments with in vitro perfusion of this isolated vascular bed (Crossley, unpublished results). The CAM also shows a limited vasoconstriction after $\alpha$-adrenergic stimulation with phenylephrine. Therefore, systemic release of catecholamines (Table 3) during hypoxia in late-stage embryonic chickens would result in a vasodilation of the CAM vessels via $\beta$-adrenoceptors while producing a vasoconstriction in some of the intraembryonic vessels via $\alpha$-adrenergic receptors. With an estimated CAM blood flow of $20-50 \%$ of total cardiac output $(2,18,21,26,27)$, CAM vasodilation could account for the global hypotension that occurred during hypoxia. Interestingly, the hypoxic pressure response to mild and moderate levels $\left(15\right.$ and $\left.10 \% \mathrm{O}_{2}\right)$ was much less accentuated in internally pipped embryos (day 20). Taking into account that effective lung ventilation starts on day 20, the differences in responses to mild and moderate hypoxia could be attributed to a transient resetting of the sensitivity to hypoxia. The change in function of the cholinergic pressure control system from a hypoxic vasoconstriction (up to day 20) to a vasodilation (on day 21) may also contribute to the different response found on day 20.

Adrenergic activation: neural vs. humoral contribution. In fetal sheep, adrenergic stimulation brought about by acute hypoxia is made up of a combination of increased nervous adrenergic drive and adrenal release of catecholamines $(7,9,14,17)$. Both mechanisms are possible in embryonic chickens given the effects of $\alpha$-blockade (Fig. 4) and the increased titers of catecholamines in the plasma during hypoxia (19). However, using field stimulation, release of norepinephrine from sympathetic terminals has been shown to occur only during the last day of incubation (22). This suggests that in embryonic chickens the humoral adren- ergic response is of greater importance during hypoxia than the neural adrenergic response. In an effort to isolate the origin of the catecholamine response to hypoxia in embryonic chickens, $6-\mathrm{OH}$ was used to eliminate the neural originating catecholamines. Unfortunately, complete chemical sympathectomy with $6-\mathrm{OH}$ was not fully achieved in this work given that the effects of tyramine were lowered but not abolished. Thus conclusions based on the response to hypoxia after 6-OH treatment must be viewed with caution. Acknowledging this consideration, the hypoxic pressure response of day 20 embryos was altered by treatment with 6-OH (Table 5). Thus day 20 embryonic chickens release catecholamines from sympathetic terminals to maintain arterial pressure during hypoxia challenges. Therefore, the sympathetic terminals are capable of releasing catecholamines during hypoxia before they were previously suggested to be functional and may contribute to the pressure response on this day of incubation (22). To further clarify the source of the hypoxic catecholaminergic and muscarinic response in embryonic chickens, hexamethonium, an antagonist of nicotinic receptors, was administered. Hexamethonium blocks autonomic transmission at the ganglionic level of both cholinergic and adrenergic fibers. Thus it blocks the centrally mediated release of catecholamines from adrenergic nerve terminals and adrenal glands. Few changes in the cardiovascular response to hypoxia were evident after hexamethonium, with those effects seen mimicking the effects previously determined with atropine. This indicates that hexamethonium did not affect the adrenergic humoral response. Thus the adrenergic response was triggered by the direct action of low oxygen on the chromaffin cells of the adrenal tissue as well as sympathetic terminals and did not involve an autonomic nervous system-originating reflex.

\section{Perspectives}

Upon exposure to hypoxia, chicken embryos display both similar features and unique regulatory mechanisms compared with fetal sheep, the standard model in the study of perinatal cardiovascular regulation. Embryonic chickens exhibit a greater bradycardic response to reduced $\mathrm{O}_{2}$ levels than do fetal sheep at a corresponding developmental age (based on percentage of gestation) (13). In chickens, the autonomic nervous system seems to play only a limited role, with the direct effect of oxygen levels on the heart as well as other local systems dominating the response to hypoxia. A contributing factor to the differing hypoxic heart rate and arterial pressure responses between these species may be the presence of a greater resistance to oxygen diffusion in chicken embryos. In chickens, this larger resistance to gas flux (the eggshell through pores and the shell membranes) causes a low oxygen saturation of systemic blood (as low as 27\%) (25) compared with the $60 \%$ saturation reported in fetal sheep (15). Thus small changes in environmental $\mathrm{O}_{2}$ could result in larger changes in blood $\mathrm{Po}_{2}$ levels in 
chicken embryos producing a greater sensitivity to hypoxic exposure compared with fetal sheep.

Fetal sheep and embryonic chickens also differ in terms of their regulation of the hypoxic response. This was evident in the hypoxic hypotension of embryonic chickens instead of the reflex hypertension displayed by fetal sheep (13). This difference may be due to the existence of an important $\beta$-adrenergic receptor-dependent vasodilation of the CAM vasculature in chickens, a character not shared by its mammalian analog, the placenta, which has been found to be catecholamine insensitive $(5,28)$. This CAM dilation in chickens may counteract the $\alpha$-adrenergic-stimulated vasoconstriction found in both species during hypoxia derived from catecholamines originating from the adrenal medulla and sympathetic nerves in later embryonic development. Thus this possibly accounts for the differing hypoxic pressure responses between embryonic chickens and fetal sheep. Furthermore, there is evidence that cholinergic receptors are involved in the vascular control in chicken embryos, which again differs from fetal sheep. Thus the redistribution of cardiac output during hypoxia is similar between chicken and sheep (18); however, it appears to be achieved via differing mechanisms. This implies that the maintenance of blood flow to the heart, the brain, and exchange organ (CAM or placenta) is not dependent on the same regulatory mechanisms between these species.

We are greatly indebted to G. Rydgren for catecholamine analysis. The work was supported by National Science Foundation Grant 1BN-9616138 to W. W. Burggren.

\section{REFERENCES}

1. Altimiras J and Crossley DA II. Control of blood pressure mediated by baroreflex changes of heart rate in the chicken embryo (Gallus gallus). Am J Physiol Regul Integr Comp Physiol 278: R980-R986, 2000.

2. Ar A, Girard H, and Rodeau JL. Oxygen uptake and chorioallantoic blood flow changes during acute hypoxia and hyperoxia in the 16-day chicken embryo. Respir Physiol 83: 295-312, 1991.

3. Bennett T. Peripheral and autonomic nervous systems. In: Avian Biology, edited by Farner DS, King JS, and Parkes KC. New York: Academic, 1974, p. 1-77.

4. Bennett T and Malmfors T. Regeneration of the noradrenergic innervation of the cardiovascular system of the chick following treatment with 6-hydroxydopamine. J Physiol 242: 517-532, 1974.

5. Berman W Jr, Goodlin RC, Heymann MA, and Rudolph AM. Effects of pharmacologic agents on umbilical blood flow in fetal lambs in utero. Biol Neonate 33: 225-235, 1978.

6. Burnstock G. Local mechanisms of blood flow control by perivascular nerves and endothelium. J Hypertens 8: S95-S106, 1990.

7. Cheung CY. Fetal adrenal medulla catecholamine response to hypoxia-direct and neural components. Am J Physiol Regul Integr Comp Physiol 258: R1340-R1346, 1990.

8. Cohen HL. Development of autonomic innervation in mammalian myocardium. In: Developmental Neurobiology of the Autonomic Nervous System, edited by Gootman PM. Clifton, NJ: Humana, 1986, p. 159-191.

9. Cohen WR, Piasecki GR, Cohn HE, Young JB, and Jackson BT. Adrenal secretion of catecholamines during hypoxemia in fetal lambs. Endocrinology 114: 383-390, 1984.
10. Crossley DA II and Altimiras J. Ontogeny of autonomic control of cardiovascular function in the domestic chicken Gallus gallus. Am J Physiol Regul Integr Comp Physiol 279: R1091R1098, 2000.

11. Dawes GS, Johnston BM, and Walker DW. Relationship of arterial pressure and heart rate in fetal, new-born and adult sheep. J Physiol 309: 405-417, 1980.

12. Giussani DA, Spencer JAD, and Hanson MA. Fetal cardiovascular reflex responses to hypoxaemia. Fetal Mat Med Rev 6: 17-37, 1994.

13. Giussani DA, Spencer JAD, Moore PJ, Bennet L, and Hanson MA. Afferent and efferent components of the cardiovascular reflex responses to acute hypoxia in term fetal sheep. J Physiol 461: 431-449, 1993.

14. Iwamoto HS. Cardiovascular effects of acute fetal hypoxia and asphyxia. In: Fetus and Neonate. Physiology and Clinical Applications, edited by Hanson MA, Spencer JAD, and Rodeck CH. Cambridge: Cambridge University Press, 1993, p. 197-214.

15. Jensen A and Berger R. Regional distribution of cardiac output. In: Fetus and Neonate. Physiological and Clinical Applications, edited by Hanson MA, Spencer JAD, and Rodeck CH. Cambridge: Cambridge University Press, 1993, p. 23-74.

16. Kummer $\mathbf{W}$ and Haberberger R. Extrinsic and intrinsic cholinergic systems of the vascular wall. Eur J Morphol 37: 223226, 1999.

17. Lewis AB and Sischo W. Cardiovascular and catecholamine responses to hypoxemia in chemically sympathectomized fetal lambs. Dev Pharmacol Ther 8: 129-140, 1985.

18. Mulder ALM, Van Golde JC, Prinzen FW, and Blanco CE. Cardiac output distribution in response to hypoxia in the chick embryo in the second half of the incubation time. J Physiol 508: 281-287, 1998.

19. Mulder ALM, Van Golde JC, Van Goor AAC, Giussani DA, and Blanco CE. Developmental changes in plasma catecholamine concentrations during normoxia and acute hypoxia in the chick embryo. J Physiol 527: 593-599, 2000.

20. Mulder ALM, Van Goor CA, Giussani DA, and Blanco CE. $\alpha$-Adrenergic contribution to the cardiovascular response to acute hypoxemia in the chick embryo. Am J Physiol Regul Integr Comp Physiol 281: R2004-R2010, 2001.

21. Mulder ALM, Van Golde JC, Prinzen FW, and Blanco CE. Cardiac output distribution in the chick embryo from stage 36-45. Cardiovasc Res 34: 525-528, 1997.

22. Pappano AJ, and Löffelholz K. Ontogenesis of adrenergic and cholinergic neuroeffector transmission in chick embryo heart. J Pharmacol Exp Ther 191: 468-478, 1974.

23. Segar JL, Hajduczok G, Smith BA, Merrill DC, and Robillard JE. Ontogeny of baroreflex control of renal sympathetic nerve activity and heart rate. Am J Physiol Heart Circ Physiol 263: H1819-H1826, 1992.

24. Tazawa $\mathrm{H}$. Effect of $\mathrm{O}_{2}$ and $\mathrm{CO}_{2}$ in $\mathrm{N}_{2}, \mathrm{He}$, and $\mathrm{SF}_{6}$ on chick embryo blood pressure and heart rate. J Appl Physiol 51: 10171022, 1981.

25. Tazawa H and Hou PCL. Avian cardiovascular development. In: Cardiovascular Development: From Molecules to Organisms, edited by Burggren WW and Keller B. Cambridge, UK: Cambridge Univ. Press, 1997, p. 193-210.

26. Tazawa H, Lomholt JP, and Johansen K. Direct measurements of allantoic blood flow in the chicken, Gallus domesticus. Responses to alteration in ambient temperature and $\mathrm{PO}_{2}$. Comp. Biochem. Physiol. 81A: 641-642, 1985.

27. White PT. Experimental studies on the circulatory system of the late chick embryo. J Exp Biol 61: 571-592, 1974.

28. Yoshimura T, Magness RR, and Rosenfeld CR. Angiotensin II and $\alpha$-agonist. I Responses of ovine fetoplacental vasculature. Am J Physiol Heart Circ Physiol 259: H464-H472, 1990.

29. Zaimis E. The usefulness of the immunosympathectomized animal in pharmacological studies. In: Nerve Growth Factor and Its Antiserum, edited by Zaimis E and Knight J. London: University College London, 1972, p. 185-200. 